\title{
THE ULTRAVIOLET BACKGROUND (INTERGALACTIC GAS, THE GALAXY, AND SUBCOSMIC RAYS)
}

\author{
V. G. KURT and R. A. SUNYAEV \\ Academy of Sciences, U.S.S.R. and Sternberg Astronomical Institute
}

\begin{abstract}
Observations. - A survey is made of observations of the background radiation at UV wavelengths from above the atmosphere. Sources of the background radiation and ways of determining the extragalactic component of the background are discussed. Future observations are also discussed.

(2) Cosmology. - Limits to the properties of the intergalactic gas follow from observations of the UV background. The problem of detecting galaxies at early stages in their evolution is considered.

(3) The Galaxy.-Observations and theoretical estimates are given for the integrated brightness of the Galaxy at UV wavelengths beyond the Lyman- $\alpha$ line. Also discussed are the nature of the Lyman- $\alpha$ emission from the Milky Way and the principal ways of constructing the luminosity function of stars in the Galaxy from observations of the integrated UV spectrum.

(4) Subcosmic Rays in the Interstellar Medium. - Fast neutral excited hydrogen atoms can be formed from charge-exchange interactions between subcosmic-ray protons and neutral interstellar gas. Upper limits are given to the energy density of subcosmic rays having $E \sim 100 \mathrm{keV}$.

(5) Limits on the Background Radiation in the range $<912$ A. - The distribution of neutral hydrogen in the peripheries of galaxies allows limits to be obtained for the flux of metagalactic ionising radiation. The heating and ionisation of the interstellar medium by $\mathrm{X}$-rays is considered.
\end{abstract}

\section{Introduction}

It is now relatively easy to make observations in the near ultraviolet region of the spectrum (1000-3000 $\AA$ ) from space vehicles above the atmosphere. Particularly great progress has been made in the study of the spectra of stars of early spectral type with high spectral resolution $(\Delta \lambda \sim 1 \AA)$. This is the work of Carruthers, Morton, Smith, Stecher, and others.

Below we discuss observations of the ultraviolet background radiation with lower spectral resolution $(\sim 100 \AA)$ and the conclusions which can be drawn from the existing observational data concerning

(a) the density and temperature of the intergalactic gas which make it possible to determine the mean density of matter in the Universe (Kurt and Sunyaev, 1967a, b).

(b) the integrated ultraviolet radiation of our Galaxy (Kurt and Sunyaev, 1967c; Kurt and Dostovalov, 1968).

(c) the energy density of subcosmic rays in our Galaxy (Kurt and Sunyaev, 1968).

In addition we discuss indirect methods of determining the flux of background UV radiation in the wavelength range shorter than $912 \AA$ from the distribution of neutral hydrogen in the peripheries of galaxies (Sunyaev, 1969a). It is clearly impossible to observe this radiation from the solar system but it has an important influence on the properties of the interstellar medium, such as its heating and its state of ionisation (Sunyaev, 1969b).

In this survey we propose a series of new experiments which will give important information about the properties of the intergalactic gas, the overall properties of the stellar population and the interstellar medium. 


\section{The Observations}

In the observations in the UV region, photon counters and photomultipliers with a field of view $1-10^{\circ}$ have been used. In these observations the measured signal must be separated from background noise due to cosmic rays and the dark current of the photoelectric apparatus. The main contribution to the observed signal in near UV and in the visible region of the spectrum results from the integral effect of stars in our Galaxy even in the direction of the galactic pole and from zodiacal light. Therefore in future observations, we must use apparatus with a smaller field of view to obtain a smaller contribution from stars and electronic systems to decrease the contribution from charged particles. In Figure 1 we present the observational data in the range $5000-1000 \AA$. In the range $<2000 \AA$, there seems to be a possibility of obtaining data without the influence of galactic stars at high galactic latitudes. In this spectral region, the principle contribution comes from early-type stars the number of which, per square degree drops rapidly with increasing temperature. This question has been discussed in detail by us (Kurt and Sunyaev, 1967b) for the spectral range $1300 \AA$ where the principal role is played by A-B stars. If the field of view of the apparatus is $\sim 0.1$ square degree, such stars result in a background of less than $10^{-23}-10^{-22}$ erg $\mathrm{cm}^{-2}$ ster $^{-1} \mathrm{~Hz}^{-1}$ at high galactic latitudes. According to our calculations, this value is of the same order as the intensity expected from interplanetary space and the extragalactic background from galaxies. For similar observations in the visible region of the spectrum, the background results from the sum of stars of spectral class similar to the Sun and zodiacal light, the colour temperature of which is similar to that of the Sun, and the separation of the non-stellar component is extremely complicated.



Fig. 1 
According to the new data of Lillie (1969), the density of radiation near the Sun is $5 \times 10^{-17} \mathrm{erg} \mathrm{cm}^{-3} \AA^{-1}$ in the range $2000-5600 \AA$ which corresponds to $1.5 \times 10^{-7}$

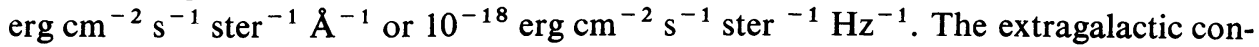
tribution of this radiation at $4100 \AA$ is less than $10^{-20} \mathrm{erg} \mathrm{cm}^{-2} \mathrm{~s}^{-1} \mathrm{ster}^{-1} \mathrm{~Hz}^{-1}$. This result is one order of magnitude lower than that of Roach and Smith (1968) and equal to that expected from the integrated effect of galaxies. This implies that any evolution of the brightness of galaxies with cosmological epoch must be small.

We have also observed the short wavelength range 1050-1180 $\AA$ with a field of view $\sim 20^{\circ}$. The observed value of the background intensity, $7 \times 10^{-21} \mathrm{erg} \mathrm{cm}^{-2}$ $\mathrm{s}^{-1} \mathrm{ster}^{-1} \mathrm{~Hz}^{-1}$, clearly gives an upper limit to the metagalactic radiation. It is possible that the main contribution to the measured signal, which is $5-10$ times greater than the background due to cosmic rays, is the result of stars with maxima in the range $2000 \AA$. In this range, the quantum efficiency is less than or equal to $10^{-4}$, which is not sufficient to reduce the background due to stars in the range $2000 \AA$.

\section{The Background Radiation from Intergalactic Space for Wavelengths Shorter than $912 \AA$}

Direct observations of the background radiation from intergalactic space in the range 912-100 $\AA$ is in principle impossible because it is completely absorbed by neutral hydrogen in our Galaxy. At present we have only observations in the soft X-ray region 44-70 $\AA$ (Bowyer et al., 1968; Henry et al., 1968) and the region close to Lyman $\alpha$ (1225-1340 $\AA$ ) which has been discussed above. The observed soft X-ray intensity is $0.5 \times 10^{-24}<I_{v}<5 \times 10^{-24} \mathrm{erg} \mathrm{cm}^{-2} \mathrm{~s}^{-1} \mathrm{ster}^{-1} \mathrm{~Hz}^{-1}$ about $44 \AA$; the exact value of this flux is not known because we do not know how strongly the radiation is absorbed in our Galaxy. In the range 1225-1340 $\AA$, the extragalactic background is not greater than $10^{-21} \mathrm{erg} \mathrm{cm}^{-2} \mathrm{~s}^{-1} \mathrm{ster}^{-1} \mathrm{~Hz}^{-1}$. At the present day, there is only one way of observing the background radiation for wavelengths less than $912 \AA$ which is from observations of the peripheries of galaxies in the $21-\mathrm{cm}$ line of neutral hydrogen. It is clear that as a result of interactions between the background ionising radiation ( $<912 \AA)$ and neutral hydrogen in the peripheries of galaxies, there must exist ionisation zones analogous to Strömgren spheres around hot stars. Observational techniques for observing the $21-\mathrm{cm}$ line are now highly developed and the distribution of hydrogen in the Andromeda Nebula (M31), the Magellanic clouds and at least 10 other galaxies and systems of galaxies is known. Observations have shown that the hydrogen halo extends far beyond the optical image of the galaxy (at least 1.5 times) and that neighbouring galaxies which have no connection at optical wavelengths are joined by bridges of neutral hydrogen (NGC 4631-4656). The dimensions of these bridges are very large but the number of atoms along the line of sight is much smaller than in the central regions of the galaxies; it follows that the density of hydrogen is very small and must be of the order of $3 \times 10^{-3} \mathrm{~cm}^{-3}$ or less. An ionisation front forms at the periphery of the galaxy as a result of the incident flux of ionising radiation and moves into the galaxy with velocity $V=I / n_{\mathrm{H}}$, where $I$ is the flux of radiation in quanta 
$\mathrm{cm}^{-2} \mathrm{~s}^{-1}$ and $n_{\mathrm{H}}$ is the density of hydrogen in front of the ionisation front. The ionisation front continues to move into the galaxy with this velocity as long as the optical depth of the gas behind the front is not too large, i.e.

$$
\int_{0}^{\infty} n_{e}^{2} \alpha \mathrm{d} l<I,
$$

i.e. the number of recombinations for unit time in a column of gas behind the front must not exceed the number of incident quanta ( $\alpha$ is the recombination coefficient and $n_{\mathrm{e}}$ the electron density behind the front).

If the total number of neutral and ionised particles along the line of sight does not increase with distance from the centre of the galaxy, then from the observations of the Andromeda Nebula if $I>10^{4}$ quanta $\mathrm{cm}^{-2} \mathrm{~s}^{-1}$, it is highly unlikely that there should exist a stationary Strömgren zone surrounding the neutral hydrogen halo with $n_{\mathrm{H}} \sim$ $2 \times 10^{-3} \mathrm{~cm}^{-3}$ and $l \sim 3 \mathrm{kpc}$. That is, if $I>10^{4}$ quanta $\mathrm{cm}^{-2} \mathrm{~s}^{-1}$, which corresponds to $I_{v}>10^{-23} \mathrm{erg} \mathrm{cm}^{-2} \mathrm{~s}^{-1} \mathrm{ster}^{-1} \mathrm{~Hz}^{-1}$ in the range $912 \AA<\lambda<304 \AA$, the time scale for ionizing the halo be less than $10^{15} \mathrm{sec}$, which is less than the time scale of the galaxy. Similar calculations may be made for other galaxies. It is clear that even if we have a small flux of ionising radiation, a stationary or moving ionisation front must exist somewhere in the distant periphery of the galaxy and must result in a sharp boundary to the distribution of neutral hydrogen. We also note that if the ionising flux with $\lambda<912 \AA$ were greater than $I_{v} \sim 4 \times 10^{-21} \mathrm{erg} \mathrm{cm}^{-2} \mathrm{~s}^{-1} \mathrm{ster}^{-1} \mathrm{~Hz}^{-1}$, the neutral hydrogen in the plane of our Galaxy in the region of the Sun could be completely ionised $\left(n_{\mathrm{H}} l \sim 2.5 \times 10^{20} \mathrm{~cm}^{-2} ; n_{\mathrm{H}} \sim 0.1 \mathrm{~cm}^{-3}\right)$ (Sunyaev, 1969a, b). Therefore it appears that neutral hydrogen in the peripheries of galaxies is a more sensitive method of detecting the ultraviolet background radiation than existing experiments in the range $\lambda>912 \AA$.

\section{The Heating and Ionisation of the Interstellar Gas by UV and soft X-Ray Radiation}

The photons of the soft X-ray background with wavelengths around $40 \AA$ which have been detected (Bowyer et al., 1968; Henry et al., 1968), heat and partially ionise the interstellar gas (Sunyaev, 1969b), influence the physical conditions in the interstellar gas and, in particular, hinder the formation of clouds of neutral hydrogen in the peripheries of galaxies and in the regions between spiral arms - wherever $n_{\mathrm{H}} \sim 10^{-2}$ $\mathrm{cm}^{-3}$. This is an example, similar to the relict radiation, of how the background radiation can influence the physical conditions in the Galaxy.

Pikel'ner (1967) has considered the heating and partial ionisation of the interstellar gas by subcosmic rays. It is considered that the subcosmic rays are injected from supernova explosions. Calculations on the explosion of supernovae carried out by V.S. Imshennyk and his co-workers have shown that a significant part of the energy is emitted as ionising radiation in the region $200-30 \AA$. This burst of radiation heats 
and ionises the interstellar gas to a distance of hundreds of parsecs from the supernovae; the relaxation time is very large. If we suppose that in the interstellar medium $n_{\mathrm{e}} \sim 3 \times 10^{-3} \mathrm{~cm}^{-3}$ and $T_{\mathrm{e}} \sim 10^{2} \mathrm{~K}$, then the recombination time turns out to be of the order of $t \sim 1 / \alpha n_{\mathrm{e}} \sim 10^{14} \mathrm{sec}$. Comparison of this time with the frequency of supernova outbursts in the Galaxy $\left(t \sim 10^{9}-10^{10} \mathrm{sec}\right)$ shows that the UV ionising radiation must have a significant influence on the properties of the interstellar medium. This conclusion is confirmed by the existence of a large number of X-ray sources with spectra which rise sharply in the ultraviolet, additional to which normal stars may be powerful sources at about $100 \AA$ because of the existence of coronae similar to the Sun's.

\section{Cosmological Aspects of Measurements of UV Radiation}

\section{A. INTERGALACTIC GAS}

It is well known that if the mean density of matter in the Universe exceeds its critical value $\left(\varrho_{\mathrm{cr}}=\left(3 H_{0}^{2}\right) /(8 \pi g)=2 \times 10^{-29} \mathrm{~g} \mathrm{~cm}^{-3}\right)$, the Universe is closed and the expansion observed at the present day must eventually reverse to a contracting phase. If $\varrho<\varrho_{\mathrm{cr}}$, the Universe is open. The matter contained in galaxies (i.e. visible matter) and radiation imply $\varrho \ll \varrho_{\text {cr }}$ and is insufficient to close the Universe. In principle, it is possible that there exists a diffuse intergalactic gas with density $\varrho \sim \varrho_{\mathrm{cr}}$ which determines the dynamics of the Universe. The present observational data at radio $(\lambda 21 \mathrm{~cm})$ and soft $X$-ray wavelengths (44-60 $\AA$ ) set limits to the permissible range of temperature of the intergalactic gas of $10^{4}-3 \times 10^{6} \mathrm{~K}$ if $\varrho \gtrsim \varrho_{\mathrm{cr}}$. If the gas has such a density and its temperature lies in this range, it radiates principally in the ultraviolet. Therefore measurements of the intergalactic ultraviolet background can give information about the density and temperature of the intergalactic gas and the past history of the Universe. Measurements in the range 1225-1340 $\AA$ have enabled upper limits to be set to the flux in the intergalactic redshifted Lyman $\alpha$ line. It was shown (Kurt and Sunyaev, 1967a, b) that these measurements set an upper limit of $\varrho<5 \varrho_{\mathrm{cr}}$ for any temperature of the intergalactic gas and $\varrho<\varrho_{\mathrm{cr}}$ for the narrower temperature interval $3 \times 10^{4}>T>10^{4} \mathrm{~K}$. The indirect method of determining the UV background described above $(\lambda<912 \AA) \mathrm{im}$ plies that for any temperature $\varrho<0.3 \varrho_{\mathrm{cr}}$. It is clear, however, that we must continue these direct observations in the UV region of the spectrum with higher sensitivity. Measurements in the spectral region $\lambda<1216 \AA$ will enable the separation of the contributions from Lyman $\alpha$ lines, the continuous hydrogen spectrum and the He II $304 \AA$ line, all of which are displaced due to the effect of the cosmological expansion.

\section{B. OBSERVATIONS OF THE SPECTRUM OF 3C273}

Above we discussed the possibility of observing radiation from the hot ionised gas. A much more sensitive method of measuring the density of a neutral intergalactic gas than observations of the $21-\mathrm{cm}$ line is observation of quasars in the ultraviolet region of the spectrum with $\lambda>1216 \AA$. This has been done for $3 C 9$ by Gunn and Peterson (1965) who have given an upper limit $n_{\mathrm{H}}>6 \times 10^{-11} \mathrm{~cm}^{-3}$. The upper limit to the density of neutral hydrogen which may be evaluated from absorption in the optical 
spectra of quasars may be expressed as the optical depth

$$
\tau=\frac{5 \times 10^{10}(1+z)^{2} n_{\mathrm{H}}}{\sqrt{1+\left(\varrho / \varrho_{c z}\right) z}} .
$$

For $z \ll 1$, if $\tau \gtrsim 0.3$ (which could be observed), $n_{\mathrm{H}} \gtrsim 6 \times 10^{-12} \mathrm{~cm}^{-3}$. The most convenient quasar to use is $3 \mathrm{C} 273$ with $z=0.16, m=13^{\mathrm{m}}$. It would be extremely interesting to obtain spectra of this quasar in the range 2000-912 $\AA$ and also the spectra of quasars with $z \gtrsim 2$ which would allow absorption due to intergalactic neutral helium $\mathrm{He}$ $584 \AA$ to be measured. Such spectra would also give much information about the quasars themselves. The spectrum of 3C273 in the region 1000-2000 $\AA$ is probably of the form $I_{v} \sim v^{-\alpha}$ with $\alpha \approx 0.7$ as is found in quasars with $z \sim 2$. In this case, in the UV region of the spectrum, the quasar would have intensity $\sim 10^{-25} \mathrm{erg} \mathrm{cm}^{-2} \mathrm{~s}^{-1} \mathrm{~Hz}^{-1}$ at $1300 \AA$. With a bandwidth $\Delta \lambda \sim 10 \AA$ such a flux could be observed with a telescope of diameter of about $20 \mathrm{~cm}$. There remains of course the problem of orientating the telescope on to an object which is very faint at optical wavelengths but, at the same time, anomalously bright in the UV region. 3C9 would be 20 times weaker and therefore requires a 1-m telescope with the same spectral resolution. Both of these objects are excellent candidates for observations with the OAO.

\section{YOUNG GALAXIES}

Partridge and Peebles (1967) have considered the possibility of observing the radiation from young galaxies. In their model, galaxies were born very early at a redshift of about 20-30. In this case, most of their radiation falls in the infrared region where observations are extremely difficult because of the very high background and because of the low sensitivity of the detectors.

If galaxy formation continues to redshifts $z \lesssim 1$, the radiation of such objects falls principally in the ultraviolet region of the spectrum, where observing conditions are much more favourable.

Let us consider a galaxy of the type of our Galaxy in the process of collapse before star formation takes place. In this case the release of gravitational energy is $10^{59}$ ergs and the principal part of this energy will be emitted as Lyman $\alpha$ quanta over a timescale $\sim 10^{14}-10^{15} \mathrm{sec}$. Such an object will have luminosity $\sim 10^{44}-10^{45} \mathrm{erg} \mathrm{s}^{-1}$ which will be emitted in a very narrow line of band width about $1 \AA$. Such an object can even be observed at cosmological distances. In this calculation we have not considered absorption by dust which is formed at a later stage and also assumed that all the radiation escapes from the galaxy. If we include nuclear sources of energy and suppose that in the beginning stars of early spectral type are formed, then the energy output increases by a very large factor.

\section{Ultraviolet Radiation from the Galaxy}

UV measurements from the Venus space-probes determine the radiation from the 
Milky Way in the Lyman $\alpha$ line and the continuum in the range 1225-1340 $\AA$ (Kurt and Dostovalov, 1968).

\section{A. THE RADIATION IN LYMAN $\alpha$}

In the range $1050-1340 \AA$ which includes the Lyman- $\alpha$ line, the intensity at galactic longitude $l^{\mathrm{II}}=100^{\circ}$ is approximately $2.5 \times 10^{-4} \mathrm{erg} \mathrm{cm}^{-2} \mathrm{~s}^{-1} \mathrm{ster}^{-1}$ and at $l^{\mathrm{II}}=300^{\circ}$ roughly 10 times less. A similar effect has been observed in the region $l^{\mathrm{II}}=80^{\circ}$ and $l^{\mathrm{II}}=$ $120^{\circ}$, where the intensities are very similar and equal to $5-10 \times 10^{-5} \mathrm{erg} \mathrm{cm}^{-2} \mathrm{sec}^{-1}$ ster $^{-1}$. The characteristic property of this emission is that it strongly depends upon galactic longitude.

\section{B. CONTINUOUS EMISSION}

At the same time in the channel sensitive to the band 1225-1340 $\AA$ (i.e. without Lyman $\alpha$ ) the intensity of the Milky Way was equal to only $10^{-6} \mathrm{erg} \mathrm{cm}^{-2} \mathrm{ster}^{-1}$. This radiation is the result of the population of hot stars.

We now have estimates of the diffuse UV radiation from stars both from studies of experimental spectra and from theoretical model stellar atmospheres. In the spectral region 1000-1500 $\AA$, the calculated values of the intensity exceed the observed values by a factor of about 10 . Such a discrepancy could be explained in the following ways:

(1) An abrupt 'cut-off' in the spectra of stars in the range $\lambda<1500 \AA$ as has been observed in stars of earlier spectral type than A0. One could suppose that the effective temperature of A and B type stars in the ultraviolet is $2000-3000^{\circ}$ less than $T_{\text {eff }}$ in the visual region, which would result in a decrease in intensity by a factor of 10 .

(2) Blanketing of absorption lines in the range 1225-1340 $\AA$. The equivalent width grows rapidly with decreasing stellar temperature. The radiation from the Milky Way in this spectral region consists principally of stars of type B5-A5, where the strongest absorption lines are C II, C III, Si III, S II (Morton, and his co-workers) and also Lyman $\alpha$. Calculations for O5, B0 and B4 stars and also spectra obtained in the U.S.A. from rockets have enabled the background spectrum due to stars to be estimated on different assumptions about their luminosity function and interstellar absorption of UV radiation. At the present day, we know the standard luminosity function for nearby stars within a distance of $10 \mathrm{pc}$. For observations in the range 1000-2000 $\AA$ absorption by interstellar dust makes it possible to observe stars within a sphere of about $300 \mathrm{pc}$. If the bandwidth of the spectrum considered is about $100 \AA$ wide, the contribution at different wavelengths is determined by stars of a very narrow class of spectral types. This makes it possible to improve the luminosity function for O-F stars.

\section{Subcosmic Rays}

Non-resonant charge-exchange interactions of the proton component of subcosmic rays

$$
P+H \rightarrow H(2 p)+P
$$


and excitations due to fast neutral atoms with neutral interstellar hydrogen

$$
H+H \rightarrow H(2 p)+H
$$

result in the formation of excited atoms having large velocities. These atoms radiate Lyman $\alpha$ quanta with wavelength shifted because of the Doppler effect. In the region of interest to us (1225-1340 $\AA$ ) radiation comes from particles with energy between $26 \mathrm{keV}$ and several $\mathrm{MeV}$. The main contribution results from particles with energy less than $100 \mathrm{keV}$. This is because the cross-section for resonance and charge-exchange interactions decreases rapidly for energies greater than $100 \mathrm{keV}$. If we know an upper limit to the intensity of ultraviolet radiation, it is easy to give an upper limit to the density and energy density of subcosmic rays. These calculations were carried out by us (Kurt and Sunyaev, 1968) and we found

$$
\begin{aligned}
& n(E)<\frac{10^{-9}}{n_{\mathrm{H}}} \mathrm{cm}^{-3} \mathrm{keV}^{-1} \\
& W<5 \times 10^{-3} \mathrm{eV} \mathrm{cm}^{-3} .
\end{aligned}
$$

The above rough estimates can be improved by narrowing the spectral interval because $E=100 \mathrm{keV}$ corresponds to a maximum bandwidth of $17 \AA$. In the existing measurements the bandwidth was 10 times broader. Observations with small angular resolution perpendicular to the Galactic plane can exclude the background due to stars. It would also be very interesting to study the spectra of the background for $\lambda<1216 \AA$, where the radiation of stars is very weak.

\section{Acknowledgement}

We are very grateful to $\mathrm{M}$. Longair for his considerable help.

\section{References}

Bowyer, C. S., Field, G. B., and Mack, J. E.: 1968, Nature 217, 32.

Gunn, J. E. and Peterson, B. A.: 1965, Astrophys. J. 142, 1633.

Henry, R. C., Fritz, G., Meekins, J. E., Friedman, H., and Byram, E. T.: 1968, Astrophys. J. 153, L11. Kurt, V. G. and Dostovalov, S. B.: 1968, Nature 217, 219.

Kurt, V. G. and Sunyaev, R. A.: 1967a, JETP Letters 5, 299.

Kurt, V. G. and Sunyaev, R. A.: 1967b, Cosmic Research [Kosmicheskie issledovanija] 5, 496.

Kurt, V. G. and Sunyaev, R. A.: 1967c, Astron. J. USSR 44, 1157.

Kurt, V. G. and Sunyaev, R. A.: 1968, JETP Letters 7, 215.

Lillie, C. F.: 1969, Bull. Am. Astron. Soc. 1, 132.

Partridge, R. B. and Peebles, P. J. E.: 1967, Astrophys. J. 177, 868.

Pikel'ner, S. B.: 1967, Astron. J. USSR 44, 1915.

Roach, F. F. and Smith, L. L.: 1968, Geophys. J.15, 227.

Sunyaev, R. A.: 1969a, Astrophys. Letters 3, 33.

Sunyaev, R. A.: 1969b, Astron.J.USSR 46, 929. 